likely they are to be presented to the immune system and recognized as foreign, has been shown to be a critical factor in predicting the Structural Determinants of Neoantigen Immunogenicity for Cancer Therapy

Jason Devlin ${ }^{1}$, Sara Bobisse ${ }^{2}$, Alexandre Harari ${ }^{2}$ and Brian Baker ${ }^{1}$ ${ }^{1}$ University of Notre Dame and ${ }^{2}$ University of Lausanne, Lausanne, Switzerland

OBJECTIVES/SPECIFIC AIMS: We are exploring the structure of the interaction between an immunogenic neoantigen and a $\mathrm{T}$ cell receptor (TCR) that recognizes the neoantigen while tolerating the counterpart self antigen. No structural example exists to date of how a TCR can discriminate between a neoantigen and the self antigen. We aim to determine the structural and biophysical features that underlie the immunogenicity for this neoantigen, and the features we determine are likely to be present in other immunogenic neoantigens. Algorithms to predict the immunogenicity of neoantigens are available, but do not incorporate structural or biophysical factors. We aim to improve these methods for immunogenic neoantigen prediction by determining structural and biophysical factors that result in recognition by the immune system. METHODS/STUDY POPULATION: Recombinant protein expression, production, and purification. Protein x-ray crystallography. Biophysical proteinprotein binding experiments RESULTS/ANTICIPATED RESULTS: The $\mathrm{T}$ cell receptor (TCR) bound to the neoantigen with an affinity 15 -fold higher than the self antigen. The leucine to phenylalanine mutation occurs at position 8 of a 9 -amino acid long peptide antigen. This position is typically in the interface bound by the T cell receptor. The structures of the unbound neoantigen and self antigen showed that the mutated residue was in the TCR interface. Additionally we noted a change in the side chain position of a proximal tryptophan, potentially due to clashes with the larger phenylalanine residue. The structure of the TCR bound to the neoantigen showed that the TCR interacted with the tryptophan in the mutation-induced conformation and with the phenylalanine residue. Thus the mutation may be altering TCR binding affinity by interactions of the residue itself with the TCR, and by locking the proximal tryptophan residue in an optimal position to interact with the TCR. We are testing the contributions of each of these factors to the overall affinity change. Hydrophobicity has been linked to immunogenicity, so mutations that increase hydrophobicity compared to the self antigen are likely to be immunogenic. However, leucine and phenylalanine are similar on hydrophobicity scales. On the other hand, a side chain rotation is unlikely to represent a large energy barrier. Therefore, we hypothesize that another property of the phenylalanine, such as size or aromaticity, is driving the affinity difference. DISCUSSION/ SIGNIFICANCE OF IMPACT: Traditional forms of cancer therapy do not specifically target cancer cells, and their toxicity to healthy cells limits their effectiveness. Immunotherapy, which involves orchestrating a specific anti-cancer immune response, is now an established cancer therapy. Several forms of immunotherapy target "neoantigens," which are derived from mutated proteins in cancer, and are therefore are cancer-specific. Neoantigens represent a foothold that can allow the immune system to distinguish between cancer cells and healthy cells, and thus specifically target cancer cells for destruction while imparting no activity toward healthy cells that lack the neoantigen. Most cancer mutations that result in neoantigens arise from random passenger mutations in cancer and will be different among patients. Neoantigen-based cancer therapies are thus a precision medicine technique. The quality of neoantigens to induce an immune response (immunogenicity), which relates to how outcome of immunotherapy treatment. We are investigating, on a structural and biophysical level, features that may increase the likelihood of a neoantigen being recognized as foreign by the immune system. The structural insight we gain can be incorporated into algorithms that predict neoantigens from cancer exome sequencing for patient-specific identification of immunogenic neoantigens for immunotherapeutic intervention.

\section{TGFbeta, Early Cytokine Dysregulation, and Airway Smooth Muscle Dysfunction in Cystic Fibrosis}

Elizabeth L Kramer ${ }^{1}$, Rhonda Szczesniak ${ }^{1}$, Weiji Su${ }^{1}$, Satish Madala ${ }^{1}$, Kristin Hudock ${ }^{1}$, Cynthia Davidson ${ }^{1}$, Alicia Ostmann ${ }^{1}$,

Lauren Strecker ${ }^{1}$ and John P. Clancy ${ }^{1}$

${ }^{1}$ Cincinnati Children's Hospital Medical Center

OBJECTIVES/SPECIFIC AIMS: This study aims to first describe the unique cytokine profile and TGFbeta levels of young children with CF, then understand the pathologic effects of TGFbeta on lung function in a CF animal model. These powerful translational studies linking observations in clinical disease with a transgenic mouse model allow us a unique opportunity to investigate the role of TGFbeta in early CF lung disease. METHODS/STUDY POPULATION: Cytokine levels (TGFbeta, TNFalpha, IL-8, IL-6, HNE, and IL-1beta) in bronchoalveolar lavage fluid (BALF) from CF patients $(n=15)$ and non-CF control patients $(n=21)$ under 6 years old were determined by ELISA and Luminex assay. Tracheotomized patients without significant underlying lung disease were chosen as non-CF inflamed control patients, as they had similar levels of neutrophilic inflammation and infection as CF patients. The percentage of BAL neutrophils (\% PMNs) in each sample was assessed. The relationships between cytokines were analyzed using linear regression and principal components analysis. In animal studies, $\mathrm{CF}$ and non-CF mice ( $\mathrm{n}=4-5$ per group) were treated with intratracheal adenoviral TGFbetal vector, an empty vector control, or PBS. After one week, animals were collected; lung function, response to the bronchoconstrictor methacholine, and rescue with albuterol were measured utilizing a FlexiVent machine. Lungs were collected for histology. Immunohistochemistry for alpha-SMA was performed and pictures of all cross-sectional airways were obtained. Burden of ASM was assessed by dividing the square root of alpha-SMA stained airway smooth muscle by the basement membrane perimeter length of each airway. RESULTS/ANTICIPATED RESULTS: Patient characteristics of CF and non-CF inflamed control patients were similar in terms of age ( 3.6 yrs vs 3.3 yrs respectively, $\mathrm{p}=0.49$ ), positive BAL culture ( $13 \%$ vs $14 \%, \mathrm{p}=0.94)$, and $\%$ PMNs ( $65 \%$ vs $56 \%, \mathrm{p}=0.64)$. Despite these similarities, TGFbeta levels were 2-fold higher in $\mathrm{CF}$ versus non-CF BAL $(\mathrm{p}=0.034)$. Analysis of BAL cytokines from both patient groups showed that three principal components describe $86 \%$ of total variance across the cytokine variables. These components represent different contributions from the cytokines, with TGFbeta, IL6, and \% PMNs comprising one component of similarly regulated inflammatory markers. These components can distinguish CF versus non-CF patients with 77\% accuracy (area under the curve: 0.77$)$. TGFbeta concentrations were uniquely associated with increased IL-6 in CF samples $(r=0.74 ; p=0.0015)$ but did not demonstrate association with other cytokines. After TGFbeta exposure, CF mice demonstrated greater abnormalities 
in airway resistance than non-CF mice, with heightened response to methacholine. Importantly, this increase in airway obstruction in CF mice was reversible with albuterol treatment, indicating airway smooth muscle dysfunction as a principal driver of lung function abnormalities. Furthermore, TGFbeta induced an increased ASM burden on lung histology in both CF and non-CF mice $(\mathrm{p}<0.05)$. IL-6 levels in the BAL of CF mice showed greater increases after TGFbeta treatment compared to non-CF mice $(\mathrm{p}<0.05)$. Empty vector control treatment did not cause lung pathology. DISCUSSION/ SIGNIFICANCE OF IMPACT: Young children with CF have a unique pattern of pulmonary inflammation compared to inflamed non-CF control patients. In CF, TGFbeta pulmonary levels are uniquely associated with IL-6, a driver of ASM dysfunction in other pulmonary diseases. We followed up this clinical observation study by investigating the effect of TGFbeta on pulmonary disease in a mouse model. CF mice demonstrate increased pulmonary IL-6, airway obstruction, and ASM dysfunction after TGFbeta exposure. This study provides evidence that TGFbeta is associated with a distinct cytokine pattern that may promote ASM dysfunction in early $\mathrm{CF}$ lung disease. Understanding the mechanism of early CF pathophysiology will be critical in developing targeted therapeutics that can prevent early lung damage.

3106

\author{
The influence of early life experience on telomere length, \\ health, and behavior of domestic cats \\ Mikel Maria Delgado ${ }^{1}$, Dr. Melissa Bain and \\ Dr. Tony C.A.T. Buffington \\ ${ }^{1}$ University of California, Davis
}

OBJECTIVES/SPECIFIC AIMS: The primary objective of this research is to determine whether being hand-reared, and deprived of early maternal interaction, will affect telomere length in orphaned kittens. The secondary goal is to examine how early maternal separation impacts the health, growth and behavior of orphaned kittens. METHODS/STUDY POPULATION: Kittens were fostered through local rescue groups and shelters. We collected blood samples from 42 orphaned kittens during the first week of their lives. Due to high mortality of this population, we obtained a second blood sample at eight weeks of age from only 30 of these kittens. We collected blood samples from 12 control kittens raised with mothers at during the first and eighth weeks of life. Blood samples are currently being processed with real time quantitative PCR (qPCR) by the Real-time PCR Research and Diagnostics Core Facility at the UC Davis School of Veterinary Medicine (SVM). This includes RNA extraction, cDNA synthesis, Reference Gene Validation, and qPCR analysis. Relative telomere length (RTL) will be calculated by comparing the average telomere abundance across three samples cells with that of a reference gene (single copy number) for each sample. The resulting T/S ratio (telomere to single copy) is proportional to the average telomere length. If $\mathrm{T} / \mathrm{S}=1$, then telomere length in the sample and the reference are the same. RESULTS/ANTICIPATED RESULTS: Because telomeres show the fastest rate of shortening early in life, we predict that maternal separation will increase the rate of telomere shortening in kittens. We also predict that the telomeres of orphaned kittens will be shorter at both one week and eight weeks of age, compared to controls. DISCUSSION/SIGNIFICANCE OF IMPACT: This study will increase our understanding of early life adversity, a finding that can translate to other mammals. It will inform the practice of fostering neonatal kittens, and illuminate whether these kittens might be at higher risk than mother-reared kittens for health problems (which could be investigated in future studies). If significant telomere shortening occurs between collection periods, then future studies can take more frequent blood samples to determine what stages of early development are potentially most sensitive. If differences between groups are found, this will establish a protocol for several future research projects, such as testing whether these detrimental effects can be mitigated by environmental enrichment via activation of telomerase. Telomerase is an enzyme that appears to counteract some shortening of telomeres, and is activated by several external factors, including exercise. Thus, a logical follow up study would be developing and testing age-specific and appropriate enrichments that may activate telomerase and reduce telomere loss. Physical contact, whether human, mother, or siblings, is another possible source of telomerase activation in young kittens. Future studies also could quantify the effects of different sources of physical contact on telomere shortening. Finally, a positive finding would establish a need for longitudinal studies of the effects of early weaning on feline health and behavior and whether differences in early-life telomere lengths predict health and longevity of cats.

3409

The Power of Phenotypic Extremes in Detecting Novel Genetic Modifiers of Hemophilia

Ohad Shimshon Bentur ${ }^{1}$ and Barry S. Coller, MD ${ }^{1}$

${ }^{1}$ Rockefeller University

OBJECTIVES/SPECIFIC AIMS: 1. To identify novel genetic modifiers that result in a mild bleeding phenotype in patients with FVIII $<1 \%$. 2. To examine the feasibility of a practice model that incorporates the principles and methods for both obtaining consent for NGS and returning individual research results from the sources described above. METHODS/STUDY POPULATION: 1. We plan a 3-step approach for identifying novel genetic modifiers of hemophilia: a. Obtain samples from individuals with an extremely mild bleeding phenotype: The study will be narrowed to patients with confirmed FVIII $<1 \%$, a null mutation in the gene for FVIII, and a mild bleeding phenotype according to a detailed bleeding history. b. Identify variants that modify phenotype: Whole exome sequencing will be performed, followed by a focused analysis of genes known or suspected to be involved in thrombosis and hemostasis and prediction of variant impact using algorithms that account for conservation and deleteriousness of all variants. c. Verify the impact of novel variants in independent samples: In silico (analyze genetic databases for suspected variants), in vivo (assess bleeding in animal models of hemophilia after introducing presumed modifier variants). 2. We will employ a model for obtaining informed consent and communicating individual genetic research results and results with potential clinical impact to research participants: a. The informed consent process will be performed after potential participants read a pamphlet entitled "Genetic Research at The Rockefeller University Hospital and Center for Clinical and Translational Science." The pamphlet includes 16 questions that the potential participants are urged to ask the investigator, including, "What will you look for in my genetic information?", "Will I receive results from this study?". Potential participants will also be informed of the meaning of clinically actionable variants, either pathogenic variants related to phenotype or secondary ("incidental") findings (i.e. variants unrelated to phenotype, the knowledge of which could lead to actions that may improve health). Participants who do not want to receive 